\title{
Research Article \\ Effect of Size and Shape on the Vibrational and Thermodynamic Properties of Nanomaterials
}

\author{
R. Kumar, G. Sharma, and M. Kumar \\ Department of Physics, G.B. Pant University of Agriculture and Technology, Pantnagar 263145, India \\ Correspondence should be addressed to M. Kumar; munish_dixit@yahoo.com
}

Received 12 April 2013; Revised 12 August 2013; Accepted 17 August 2013

Academic Editor: Ahmet Z. Sahin

Copyright (C) 2013 R. Kumar et al. This is an open access article distributed under the Creative Commons Attribution License, which permits unrestricted use, distribution, and reproduction in any medium, provided the original work is properly cited.

\begin{abstract}
A simple theoretical model is developed to study the size and shape dependence of vibrational and thermodynamic properties of nanomaterials. To show the real connection with the nanomaterials we have studied Debye temperature, Debye frequency, melting entropy, and enthalpy in different shapes, namely, spherical, nanowire, and nanofilm of $\beta$-Fe, Sn, Ag, and In. The results obtained are compared with the experimental data. A good agreement between the model predictions and the experimental data supports the theory developed in the present paper.
\end{abstract}

\section{Introduction}

The bulk properties of crystals depend on their structure, but at the nanoscale, in addition to the structure, their size and shape are an important factor, which influences their properties. The most significant characteristic of materials at the nanoscale is their high surface to volume ratio, which affects their thermodynamical properties. It is now well known that the melting temperature of nanoparticles depends on their size [1]. Melting temperature depression occurs for almost all free nanoparticles [2-5] and superheating has been reported for nanoparticles embedded in other host materials [6-8]. Guisbiers [9] used a top down approach and reported a universal relation, which is particularly helpful when experiments are difficult to lead on a specific material property. To validate the relation Guisbiers [9] compared the theoretical relation with experimental data of cohesive energy of Mo and $\mathrm{W}$ nanoparticles and of activation energy of diffusion for $\mathrm{Fe}$ and $\mathrm{Cu}$ nanoparticles. Guisbiers [9] also compared his results with different models predicting the size-dependent behavior of the vacancy formulation energy. The vacancy formulation energy versus the size of a spherical gold nanoparticle has been studied [9]. It has been concluded that the models reported by earlier workers support the formulation given by Guisbiers [9]. Thus, there are relatively extensive investigations on the size dependence of melting and cohesive energy of nanocrystals. However, it has not been accompanied by the necessary investigation of size dependence of vibrational properties and thermodynamics of nanocrystals. Such investigations should depend on our understanding of the size effect of melting and cohesive properties. In particular, a complete understanding of the melting transition in nanocrystals cannot be obtained without a clear understanding of enthalpy and entropy of melting, which are important properties of melting as already discussed in detail by Zhang et al. [10] as well as Safaei and Shandiz [11]. Therefore, some efforts have been made to study the phenomenon. Zhang et al. [10] demonstrated that a simple model, free from any adjustable parameter, can be developed for the melting enthalpy and melting entropy of nanocrystals based on Mott's equation for the melting entropy and a model for the size-dependent melting temperature. The results were supported by available experimental results on metallic and organic crystals. It has also been suggested that the size dependent melting enthalpy and melting entropy for nonsemiconductor crystals can be determined by the vibrational component of overall melting enthalpy and entropy.

Qi [12] developed a model for the size, and coherencedependent cohesive energy, melting temperature, melting enthalpy, vacancy formulation energy, and vacancy concentration of nanowires and nanofilms. It was found that the variation direction (increasing or decreasing) of thermodynamic 
properties is determined by the coherent interface, and quantity of variation depends on crystal size. The lattice type sensitive model for the melting entropy and enthalpy of nanocrystals has been presented by Safaei and Shandiz [11], which is based on the model for the size dependence of the melting point. The formulations have been compared with the experimental data of In and molecular dynamic simulation results of Ag nanoparticles. Xiong et al. [1] discussed that most thermodynamic properties of nanoparticles vary linearly with $D^{-1}$ as first approximation. Thus, it seems that there exist some efforts to study the size dependence of melting entropy and enthalpy of nanoparticles. Moreover, these quantities also depend on the shape, which is a very important phenomenon of nanoscience. Thus, it is legitimate and may be useful to present a simple model, which includes the effect of shape also in addition to the size, with a less number of parameters, which is the purpose of the present paper. The theoretical model is presented in Section 2 and the results and discussion in Section 3.

\section{Theoretical Model}

It has been discussed [13] that the Debye temperature, $\theta_{\mathrm{Dn}}$, of nanomaterial is related to the Debye temperature, $\theta_{\mathrm{Db}}$, of bulk material with the following relation:

$$
\frac{\theta_{\mathrm{Dn}}}{\theta_{\mathrm{Db}}}=\left(1-\frac{N}{2 n}\right)^{1 / 2},
$$

where $N$ is the number of surface atoms and $n$ is the total number of atoms. It is well known that the Debye temperature is linearly related to the Debye frequency [14]. Thus, we can write

$$
\frac{v_{\mathrm{Dn}}}{v_{\mathrm{Db}}}=\left(1-\frac{N}{2 n}\right)^{1 / 2},
$$

where $\nu_{\text {Dn }}$ is the Debye frequency of nanomaterial and $\nu_{\mathrm{Db}}$ the Debye frequency of bulk material. Thus, it is very clear that to study the Debye temperature or Debye frequency, we need the values of $N / 2 n$ of nanomaterial. Actually, $N / 2 n$ depends on the size and shape of the nanomaterials. The method to evaluate $N / 2 n$ for different shape of nanomaterials has already been discussed by Qi [15]. According to this model, $N / 2 n$ is $2 d / D$ for spherical nanosolids with $d$ being the diameter of the atom and $D$ the diameter of spherical nanosolids. For nanowire, $N / 2 n$ is $4 d / 3 L$ with $L$ being the diameter of nanowire. For nanofilm, $N / 2 n$ is $2 d / 3 h$ with $h$ being the height (size) of the nanofilm. Thus, (1) and (2) give the relations for the size dependence of Debye temperature and Debye frequency for different type (shape) of nanomaterials. We used these relations in the present paper to study the sizeand shape-dependent $\theta_{D}$ and $\nu_{D}$ of $\beta$-Fe.

Now, we proceed to discuss the size and shape dependence of melting entropy and melting enthalpy of different type of nanomaterials. The vibrational entropy $\left(S_{\mathrm{vib}}\right)$ of melting of a bulk crystal is related to the melting temperature $\left(T_{\mathrm{mb}}\right)$ as given below $[16,17]$

$$
S_{\mathrm{vib}}=\frac{3 R}{2} \ln \left(\frac{T_{\mathrm{mb}}}{C}\right)
$$

where $C$ is constant and $R$ is called gas constant. It has been discussed $[16,17]$ that the melting of metallic crystals is mainly vibrational in nature. Thus, the vibrational entropy $\left(S_{\mathrm{vib}}\right)$ is approximately equal to the melting entropy $\left(S_{\mathrm{mb}}\right)$ and vibrational enthalpy $\left(H_{\text {vib }}\right)$ is equal to the melting enthalpy $\left(H_{\mathrm{mb}}\right)$ for bulk crystals. Thus, (3) may be rewritten as follows for bulk material [1]:

$$
S_{\mathrm{mb}}=\frac{3 R}{2} \ln \left(\frac{T_{\mathrm{mb}}}{C}\right)
$$

and for nanomaterial

$$
S_{\mathrm{mn}}=\frac{3 R}{2} \ln \left(\frac{T_{\mathrm{mn}}}{C}\right)
$$

Equations (4) and (5) give the following relation:

$$
S_{\mathrm{mn}}=S_{\mathrm{mb}}+\frac{3 R}{2} \ln \left(\frac{T_{\mathrm{mn}}}{T_{\mathrm{mb}}}\right) .
$$

Liang and $\mathrm{Li}[18]$ reported the following relation:

$$
\frac{\theta_{\mathrm{Dn}}}{\theta_{\mathrm{Db}}}=\left(\frac{T_{\mathrm{mn}}}{T_{\mathrm{mb}}}\right)^{1 / 2} .
$$

Combining (6) and (7), we get the following relation:

$$
S_{\mathrm{mn}}=S_{\mathrm{mb}}+\frac{3 R}{2} \ln \left(\frac{\theta_{\mathrm{Dn}}}{\theta_{\mathrm{Db}}}\right)^{2},
$$

or

$$
S_{\mathrm{mn}}=S_{\mathrm{mb}}+\frac{3 R}{2} \ln \left(1-\frac{N}{2 n}\right)
$$

Thus, we may write $S_{\mathrm{mn}}$ of different type (shape) of nanomaterials using the value of $N / 2 n$ as discussed above.

Spherical nanosolid:

$$
S_{\mathrm{mn}}=S_{\mathrm{mb}}+\frac{3 R}{2} \ln \left(1-\frac{2 d}{D}\right)
$$

Nanowire:

$$
S_{\mathrm{mn}}=S_{\mathrm{mb}}+\frac{3 R}{2} \ln \left(1-\frac{4 d}{3 L}\right) .
$$

Nanofilm:

$$
S_{\mathrm{mn}}=S_{\mathrm{mb}}+\frac{3 R}{2} \ln \left(1-\frac{2 d}{3 h}\right) .
$$

We used these relations in the present work. The melting enthalpy $\left(H_{\mathrm{mb}}\right)$ of a bulk metallic crystal is defined as [12]

$$
H_{\mathrm{mb}}=S_{\mathrm{mb}} T_{\mathrm{mb}}
$$

The melting enthalpy of nanomaterial may be written as

$$
H_{\mathrm{mn}}=S_{\mathrm{mn}} T_{\mathrm{mn}} .
$$




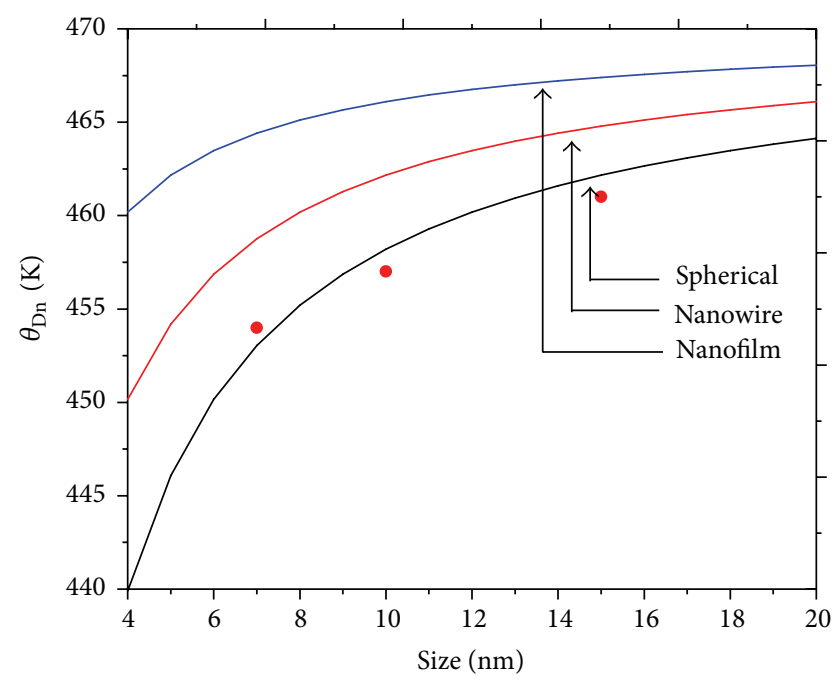

Figure 1: Size and shape dependence of Debye temperature of $\beta$-Fe using (1). The experimental data [19] are shown by for spherical.

Combining (13) and (14) gives the following relation:

$$
\frac{H_{\mathrm{mn}}}{H_{\mathrm{mb}}}=\left(\frac{S_{\mathrm{mn}}}{S_{\mathrm{mb}}}\right)\left(\frac{T_{\mathrm{mn}}}{T_{\mathrm{mb}}}\right),
$$

or

$$
\frac{H_{\mathrm{mn}}}{H_{\mathrm{mb}}}=\left(\frac{S_{\mathrm{mn}}}{S_{\mathrm{mb}}}\right)\left(\frac{\theta_{\mathrm{Dn}}}{\theta_{\mathrm{Db}}}\right)^{2}
$$

Using (1), (9), (13), and (16) gives the following relation:

$$
H_{\mathrm{mn}}=\left[H_{\mathrm{mb}}+\frac{3 R T_{\mathrm{mb}}}{2} \ln \left(1-\frac{N}{2 n}\right)\right]\left[1-\frac{N}{2 n}\right] .
$$

Thus, we can write the following relations for different type (shape) of nanomaterials.

Spherical nanosolid:

$$
H_{\mathrm{mn}}=\left[H_{\mathrm{mb}}+\frac{3 R T_{\mathrm{mb}}}{2} \ln \left(1-\frac{2 d}{D}\right)\right]\left[1-\frac{2 d}{D}\right] .
$$

Nanowire:

$$
H_{\mathrm{mn}}=\left[H_{\mathrm{mb}}+\frac{3 R T_{\mathrm{mb}}}{2} \ln \left(1-\frac{4 d}{3 L}\right)\right]\left[1-\frac{2 d}{D}\right] \text {. }
$$

Nanofilm:

$$
H_{\mathrm{mn}}=\left[H_{\mathrm{mb}}+\frac{3 R T_{\mathrm{mb}}}{2} \ln \left(1-\frac{2 d}{3 h}\right)\right]\left[1-\frac{2 d}{D}\right] .
$$

In the present paper we used these relations.

\section{Results and Discussion}

The input data $[10,11]$ required for the present work are given in Table 1. We used (1) to compute the size dependence of Debye temperature of $\beta$-Fe in different shapes, namely,

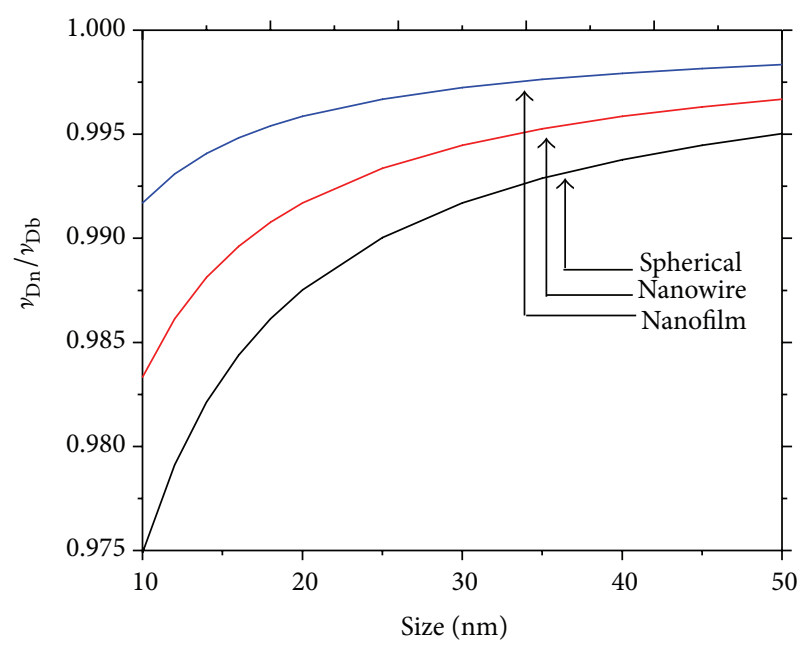

Figure 2: Size and shape dependence of Debye frequency of $\beta$-Fe using (2).

TABLE 1: Values of input parameters $[10,11]$.

\begin{tabular}{lccccc}
\hline Nanomaterials & $\begin{array}{c}d \\
(\mathrm{~nm})\end{array}$ & $\begin{array}{c}S_{\mathrm{mb}} \\
(\mathrm{J} / \mathrm{mol} \cdot \mathrm{K})\end{array}$ & $\begin{array}{c}H_{\mathrm{mb}} \\
(\mathrm{kJ} / \mathrm{mol})\end{array}$ & $\begin{array}{c}T_{\mathrm{mb}} \\
(\mathrm{K})\end{array}$ & $\begin{array}{c}\theta_{\mathrm{Db}} \\
(\mathrm{K})\end{array}$ \\
\hline$\beta$-Fe & 0.2480 & & & & 470 \\
$\mathrm{Sn}$ & 0.3725 & 14.00 & 7.08 & 1336 & \\
$\mathrm{Ag}$ & 0.2880 & 9.16 & 11.30 & 1234 & \\
$\mathrm{In}$ & 0.3291 & 7.59 & 3.28 & 429 & \\
\hline
\end{tabular}

spherical, nanowire, and nanofilm. The results obtained are reported in Figure 1. The Debye temperature is found to decrease with the decrease in the size. The trend of variation is similar in all three forms. Moreover, the Debye temperature increases for nanowire and nanofilm as compared with the spherical form of same size. The experimental data [19] are available for $\beta$-Fe (Spherical), which have been included for comparison purposes. It is found that our model predictions are in good agreement with the experimental data. We have extended the model in the form of (2) to study $\nu_{D}$ for $\beta$-Fe in different shape, namely, spherical, nanowire, and nanofilm. $v_{\text {Dn }}$ have been found to decrease with the decrease in size for all three cases with similar trend of variation as shown in Figure 2. We have further extended the model to study the size and shape dependence of melting entropy and enthalpy in the form of (10)-(12) and (18)-(20). We have considered different nanomaterials, namely, $\mathrm{Sn}, \mathrm{Ag}$, and In, because of the fact that some experimental data are available so that the model can be tested by comparison. The results obtained using (10)-(12) for melting entropy of $\mathrm{Sn}, \mathrm{Ag}$ and In in different shapes, namely, spherical, nanowire, and nanofilm, are reported in Figures 3, 4, and 5 along with the available experimental data $[10,11]$. For these materials the experimental data are available for spherical case. We have used these data $[10,11]$ for comparison purposes. It is found that our results are in good agreement with the experimental data. The melting entropy is found to decrease with decreasing the size of the materials considered in the present work. Moreover, there is increase 


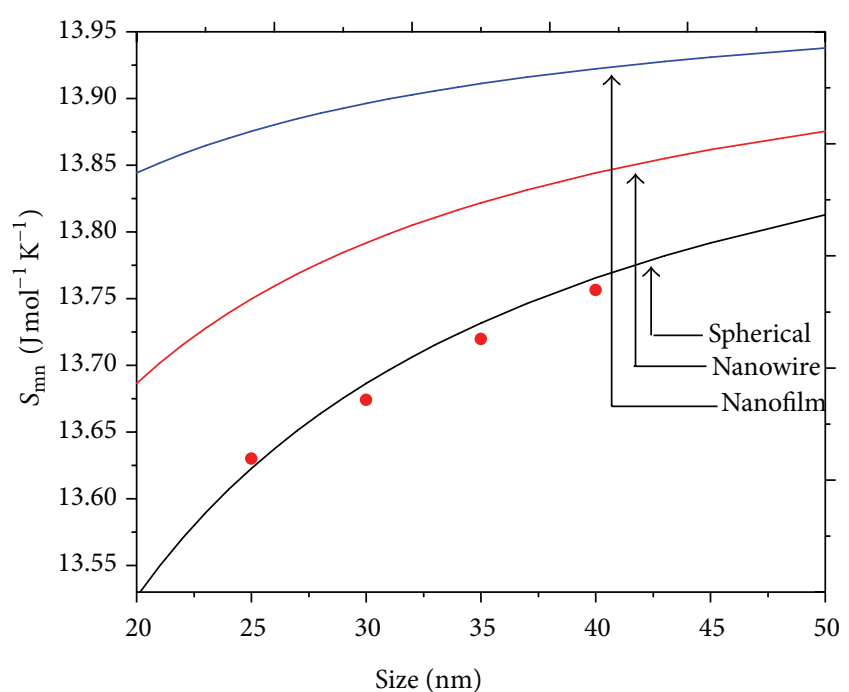

FIGURE 3: Size and shape dependence of melting entropy of Sn using (10)-(12). The Experimental data [10] are shown by $\bullet$ for spherical.

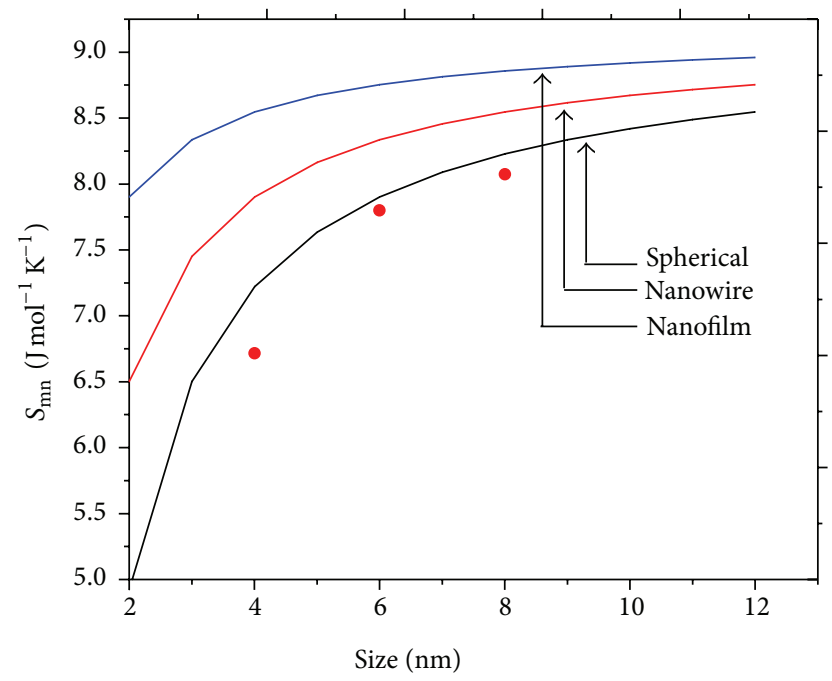

FIGURE 4: Size and shape dependence of melting entropy of Ag using (10)-(12). The experimental data [11] are shown by $\bullet$ for spherical.

in the entropy from spherical to nanowire to nanofilm for a particular size. We have also studied the size and shape dependence of melting enthalpy of $\mathrm{Sn}, \mathrm{Ag}$, and In nanomaterials in different shapes. The results obtained using (18)-(20) are reported in Figures 6, 7, and 8. There is good agreement between computed and available experimental data $[10,11]$ for spherical shape. The enthalpy is also found to decrease with the decrease in size. It should be mentioned that we are reporting the results for nanowire and nanofilm in the absence of experimental data. It may be of good interest to the persons engaged in the experimental work. By decreasing the size, increased proportion of atoms occupy surface sites which are able to move freely more than the interior atoms of nanosolids. This phenomenon leads to change in the thermodynamical properties. Enthalpy and entropy of melting

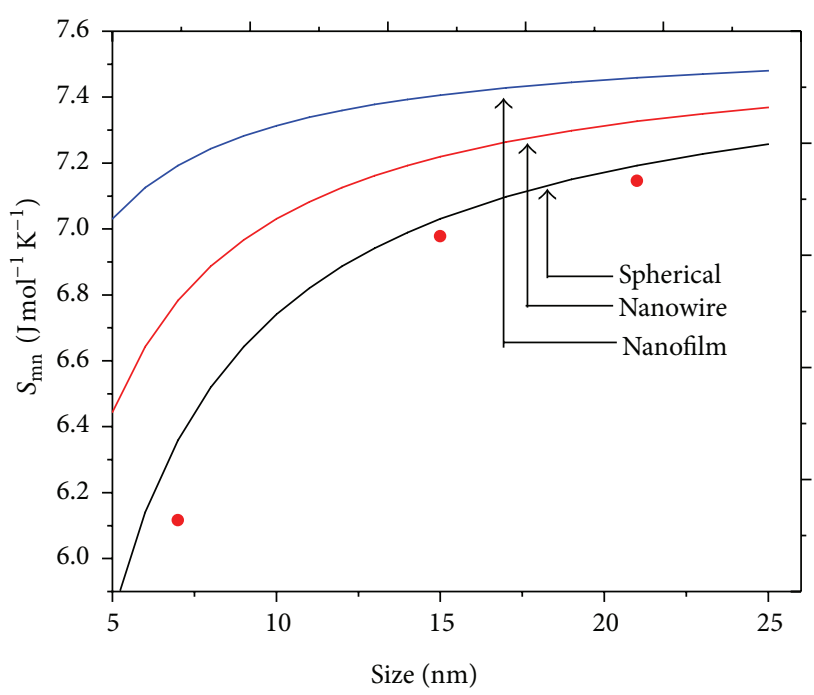

FIGURE 5: Size and shape dependence of melting entropy of In using (10)-(12). The Experimental data [11] are shown by $\bullet$ for spherical.

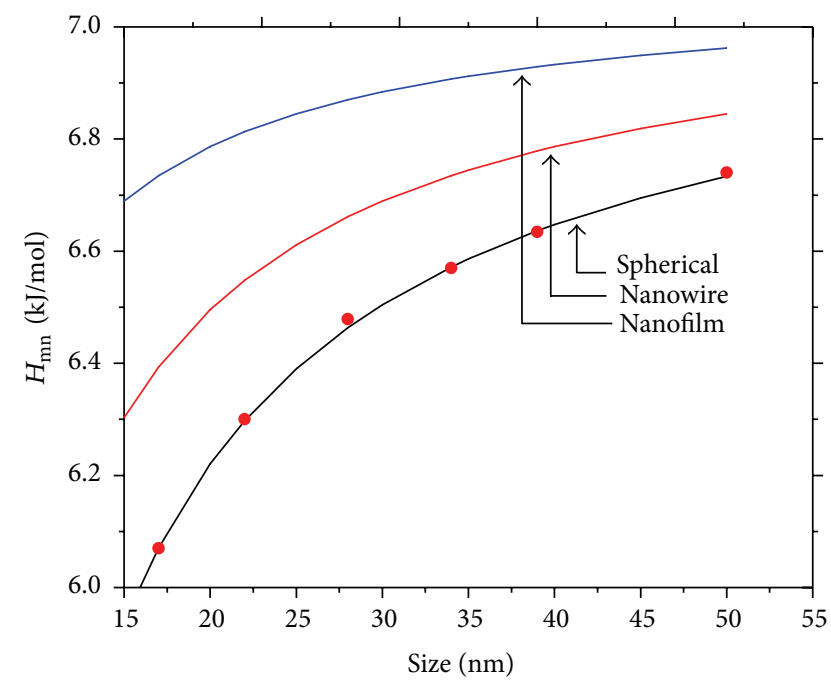

FIGURE 6: Size and shape dependence of melting enthalpy of Sn using (18)-(20). The experimental data [10] are shown by $\bullet$ for spherical.

decrease when the size decreases. The enthalpy and entropy of melting of nanoparticles may be divided into two parts: bulk quantity and surface quantity. The decrease in melting enthalpy and entropy is essentially induced by the surface contribution related to the larger surface to volume ratio and breaking bonds.

\section{Conclusion}

We have thus developed a simple theoretical model to study the size and shape dependence of Debye temperature, Debye frequency, melting entropy, and melting enthalpy. The model predictions are in good agreement with the available experimental data, which validate the proposed model. To the best 


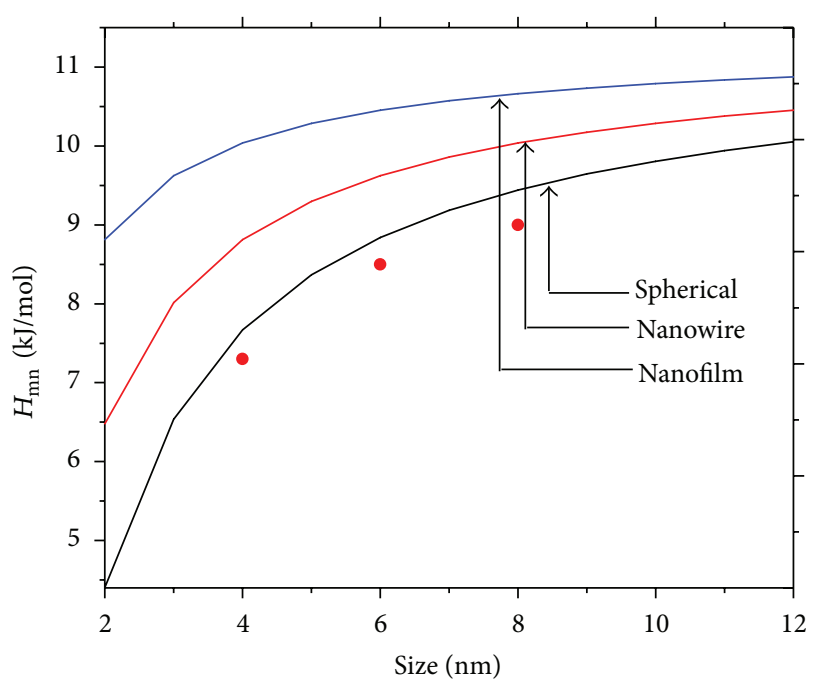

FIGURE 7: Size and shape dependence of melting enthalpy of Ag using (18)-(20). The experimental data [11] are shown by $\bullet$ for spherical.

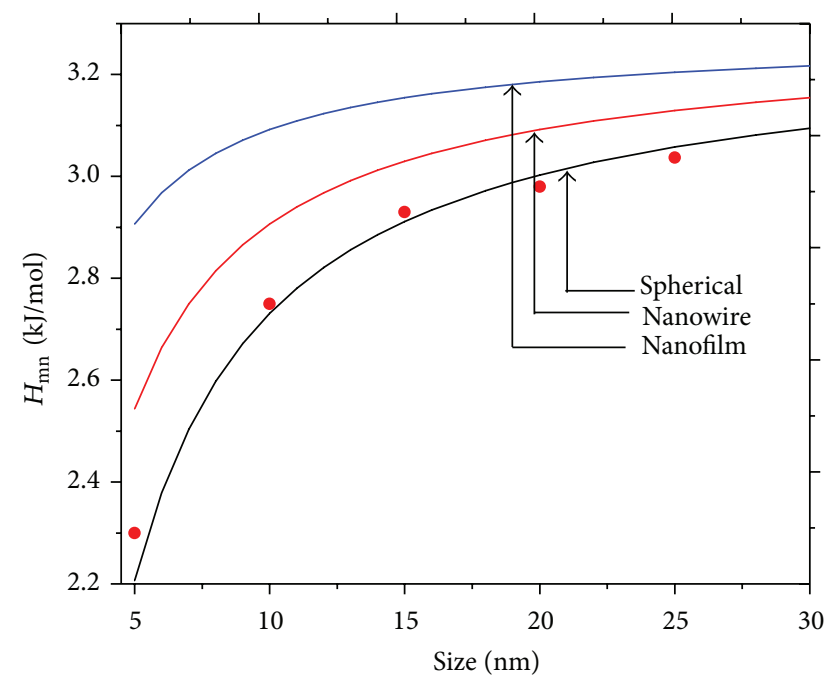

FIGURE 8: Size and shape dependence of melting enthalpy of In using (18)-(20). The experimental data [11] are shown by $\bullet$ for spherical.

of our knowledge, such simple model is not yet available in the literature to include the effect of size as well as shape. Due to the simplicity and applicability of the model, it may be extended to the other nanomaterials and may be of current interest to the researchers engaged in the study of thermodynamics of nanomaterials.

\section{References}

[1] S. Xiong, W. Qi, Y. Cheng, B. Huang, M. Wang, and Y. Li, "Universal relation for size dependent thermodynamic properties of metallic nanoparticles," Physical Chemistry Chemical Physics, vol. 13, no. 22, pp. 10652-10660, 2011.

[2] T. Ben David, Y. Lereah, G. Deutscher, R. Kofman, and P. Cheyssac, "Solid-liquid transition in ultra-fine lead particles," Philosophical Magazine A, vol. 71, no. 5, pp. 1135-1143, 1995.
[3] S. Xiao, W. Hu, and J. Yang, "Melting temperature: from nanocrystalline to amorphous phase," Journal of Chemical Physics, vol. 125, no. 18, Article ID 184504, pp. 184504-184508, 2006.

[4] P. Puri and V. Yang, "Effect of particle size on melting of aluminum at nano scales," Journal of Physical Chemistry C, vol. 111, no. 32, pp. 11776-11783, 2007.

[5] J. Eckert, J. C. Holzer, C. C. Ahn, Z. Fu, and W. L. Johnson, "Melting behavior of nanocrystalline aluminum powders," Nanostructured Materials, vol. 2, no. 4, pp. 407-413, 1993.

[6] K. Chattopadhyay and R. Goswami, "Melting and superheating of metals and alloys," Progress in Materials Science, vol. 42, no. 14, pp. 287-300, 1997.

[7] L. Gråbaek, J. Bohr, E. Johnson, A. Johansen, L. SarholtKristensen, and H. H. Andersen, "Superheating and supercooling of lead precipitates in aluminum," Physical Review Letters, vol. 64, no. 8, pp. 934-937, 1990.

[8] J. Zhong, L. H. Zhang, Z. H. Jin, M. L. Sui, and K. Lu, "Superheating of Ag nanoparticles embedded in Ni matrix," Acta Materialia, vol. 49, no. 15, pp. 2897-2904, 2001.

[9] G. Guisbiers, "Size-dependent materials properties towards a universal Equation," Nanoscale Research Letters, vol. 5, no. 7, pp. 1132-1136, 2010.

[10] Z. Zhang, X. X. Lü, and Q. Jiang, "Finite size effect on melting enthalpy and melting entropy of nanocrystals," Physica B, vol. 270, no. 3-4, pp. 249-254, 1999.

[11] A. Safaei and M. A. Shandiz, "Size-dependent thermal stability and the smallest nanocrystal," Physica E, vol. 41, no. 3, pp. 359$364,2009$.

[12] W. H. Qi, "Size- and coherence-dependent thermodynamic properties of metallic nanowires and nanofilms," Modern Physics Letters B, vol. 20, no. 30, pp. 1943-1951, 2006.

[13] R. Kumar and M. Kumar, "Effect of size on cohesive energy, melting temperature and Debye temperature of nanomaterials," Indian Journal of Pure and Applied Physics, vol. 50, no. 5, pp. 329-334, 2012.

[14] C. Kittel, Introduction to Solid State Physics, John Wiley \& Sons, New York, NY, USA, 7th edition, 1996.

[15] W. H. Qi, "Size effect on melting temperature of nanosolids," Physica B, vol. 368, no. 1-4, pp. 46-50, 2005.

[16] N. F. Mott, “The resistance of liquid metals," Proceeding of the Royal Society A, vol. 146, no. 1, pp. 456-472, 1934.

[17] A. R. Ubbelohde, Melting and Crystal Structure, Clarendon Press, Oxford, UK, 1965.

[18] L. H. Liang and B. Li, "Size-dependent thermal conductivity of nanoscale semiconducting systems," Physical Review B, vol. 73, no. 15, Article ID 153303, pp. 153303-153305, 2006.

[19] M. Hayashi, I. Tamura, Y. Fukano, and S. Kanemaki, "Mössbauer effect in small iron particles," Surface Science, vol. 106, no. 1-3, pp. 453-458, 1981. 

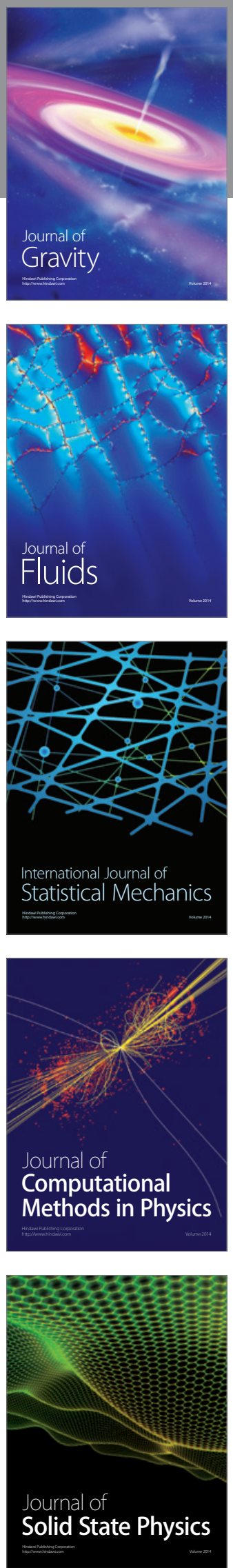

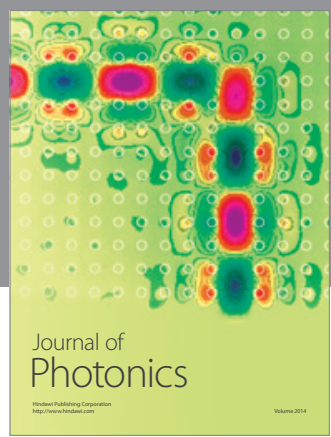

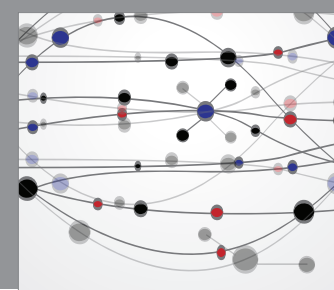

The Scientific World Journal

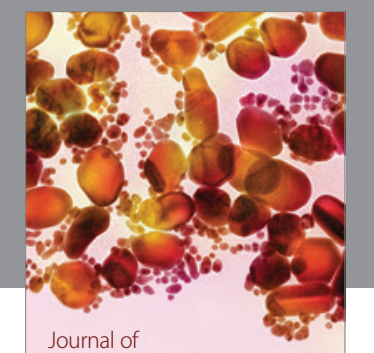

Soft Matter
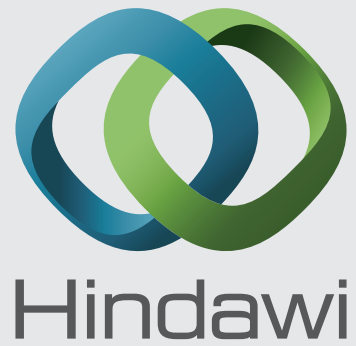

Submit your manuscripts at

http://www.hindawi.com
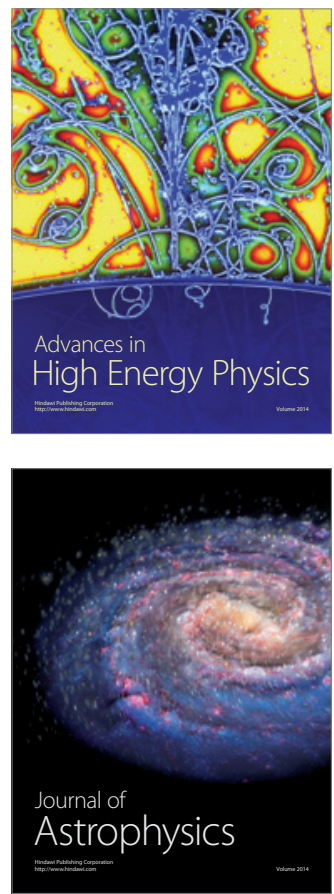
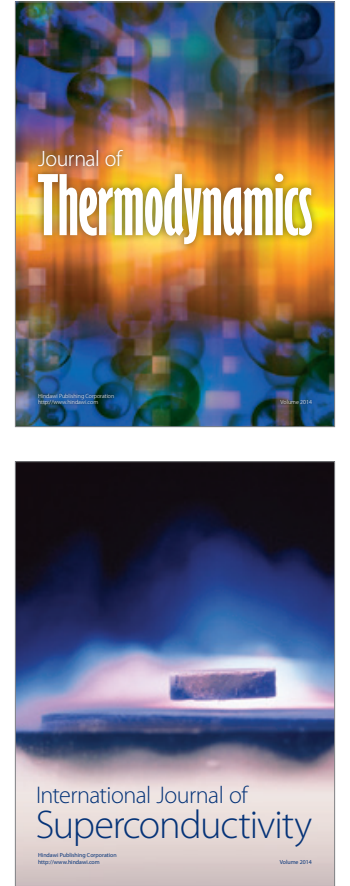
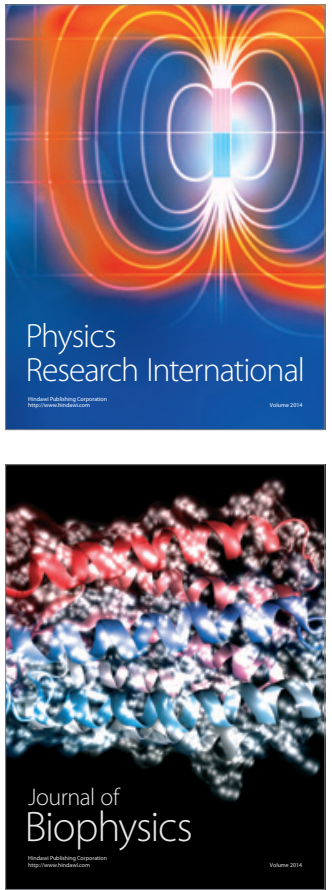
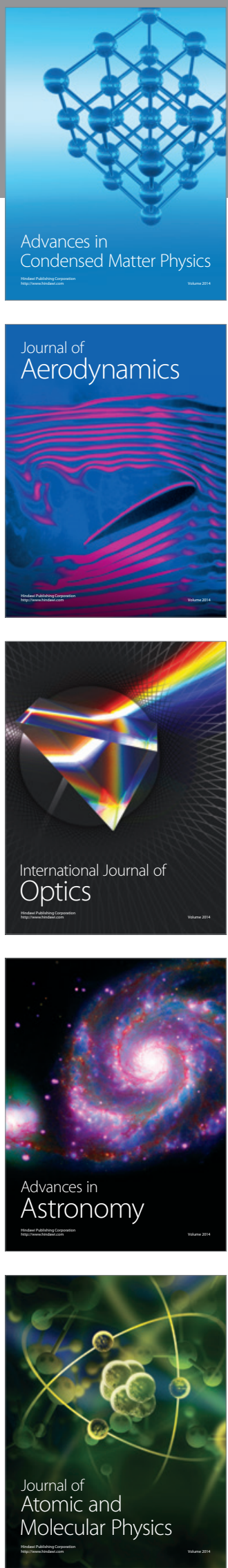